\title{
A simple collinear limit of scattering amplitudes at strong coupling
}

\author{
Gang Yang \\ Centre for Research in String Theory \\ Department of Physics, Queen Mary, University of London \\ Mile End Road, London, E1 4NS, United Kingdom
}

\begin{abstract}
Collinear limit usually provides strong constraints for scattering amplitudes. At strong coupling, collinear limit of the amplitudes in $\mathcal{N}=4 \mathrm{SYM}$ is related to the large mass limit of the corresponding $Y$ system. In this paper, we consider a special case in which all mass parameters are taken to be large, which corresponds to a multi-double-collinear limit in which a $n$-side polygon becomes pentagons. This limit provides a useful constraint for the amplitudes, in particular can be used to fix the periods part for the case of $4 K$ gluons, which is the last missing piece of full amplitudes.
\end{abstract}

\section{Introduction}

Using AdS/CFT duality, the problem of calculating scattering amplitudes at strong coupling in $\mathcal{N}=4$ super Yang-Mills theory is related to a geometric problem of computing the area of minimal surface in $A d S_{5}$ background ending on a polygonal light-like contour [1. At weak coupling, the duality between amplitudes and null polygonal Wilson loops was also found [2, 3, 4]. This duality revealed a remarkable hidden symmetry of planar scattering amplitudes, the so-called dual superconformal symmetry [1, 4, 5, 6, 7,. This is not a conventional conformal symmetry of Lagrangian but corresponds to non-trivial non-local charges, and therefore is expected to be related to the integrability of $\mathcal{N}=4$ SYM $[8,9,10,11$.

The dual conformal symmetry, via its anomaly Ward identity, can uniquely fix the four and five-point amplitudes, which are the same as the so-called BDS ansatz [12] based on the explicit perturbative calculations [13, 12, 14, 15]. However, starting at six points, the amplitudes contain a function of cross ratios which is not constrained by the dual conformal symmetry. This function is called "remainder function" 16, 17, 18, which means that it is an extra part that is not included in the BDS ansatz. The studies of the remainder function have been done both at weak coupling at two-loop level and in the strong coupling limit [19, 20, 21, 22, 23, 24, 25, 26].

The problem of calculating amplitudes at strong coupling can be solved by using the integrability of the classical world-sheet theory [23, 24, 25. The essential point is that the equations of the system can be promoted by including a spectral parameter $\zeta$ due to the integrability. The cross ratios constructed from the solutions are therefore also promoted to be functions of the spectral parameter. It then becomes possible to obtain a set of functional relations between cross ratios 
which can be organized as the so-called $Y$ system [28, 29], where $Y$ functions are the cross ratios. The boundary conditions can be nicely embedded via WKB approximation [30, 31, where the dominant behavior of $Y$ functoin at large and small $\zeta$ is given by the so-called WKB terms in which the mass parameters are related to the shape of the polygon. Finally, the main non-trivial part of the area can be given as the free energy of the $Y$ system.

The general expression of amplitudes at strong coupling can be given as [25, 27]

$$
A=A_{\text {div }}+A_{\text {BDS-like }}+A_{\text {extra }}+A_{\text {periods }}+A_{\text {free }} \text {. }
$$

By comparing to the BDS ansatz which takes the form

$$
A^{\text {BDS-ansatz }}=A_{\text {div }}+A_{\mathrm{BDS}},
$$

the reminder function at strong coupling can be given as

$$
R=\left(A_{\mathrm{BDS}-\text { like }}-A_{\mathrm{BDS}}\right)+A_{\text {extra }}+A_{\text {periods }}+A_{\text {free }} .
$$

The calculations are more tricky in the cases where the number of gluons is a multiple of four $(n=4 K)$. Such cases are special in that a world-sheet coordinate transformation appearing in the computation develops a non-trivial monodromy around infinity, which makes the calculation more complicated, in particular for the so-called cutoff part and periods part [23, 27]. In [27], it was shown that the problem can be solved by introducing two extra equations which involve non-adjacent kinematic invariants and also $T$-functions. The terms in the cutoff part that depend on the $T$-functions are defined as $A_{\text {extra, }}$, while the remaining parts are defined as $A_{\mathrm{BDS}-\text { like }}$ A conjecture for the periods part of $n=4 K$ case was also made in [27] based on a generalization of the $A d S_{3}$ result [23. However, since the case of $A d S_{5}$ is much more complicated, it's important to perform an honest calculation and check whether the conjecture is correct or not. It is calculating the periods part for the $n=4 K$ case that motivated the study of the present paper.

In this paper we study a special collinear limit of the scattering amplitudes. This limit provides a strong restriction on the amplitudes, in particular by which the periods part can be uniquely fixed by the already calculated remaining parts of the amplitude. The basic idea is very simple.

Collinear limit (or more exactly the double-collinear limit) is the limit where the momenta of two adjacent external particles become parallel. The property of amplitudes in this limit provides strong constraints for the results [32]. Particularly for the amplitudes in $\mathcal{N}=4$ SYM, the BDS ansatz already accounts for the collinear behavior of the full amplitudes [12, 17], therefore the remainder function should have a trivial behavior in the collinear limit, in the sense that a $n$-point remainder function is directly reduced to a $(n-1)$-point remainder function. It is interesting to consider a series of collinear limits so that a $n$-side polygon is finally reduced to a pentagon. Since pentagon has no non-trivial reminder function (only a constant), the $n$-point reminder function is reduced to a trivial constant in this special limit. This is the limit on which we will focus in this paper.

At strong coupling, this special limit has a nice picture in the corresponding integrable system. The structure of minimal surface is determined by a polynomial $P(z)$, where $z$ is the worldsheet coordinate. For $n$-side polygon, the degree of the polynomial is $(n-4)$. The shape of the polygon is determined by the coefficients in the polynomial that are also related to the mass parameters of the $Y$ system, which are defined as cycle integrals around two zeros of the polynomial. Taking 
one mass parameter to be infinitely large is equivalent to taking one of the zeros of the polynomial to be infinity. The corresponding picture for the minimal surface is that the a $n$-side polygon becomes a $(n-1)$-side polygon plus a decoupled pentagon (which corresponds to the decoupled zero at infinity). If all of the mass parameters are taken to be infinity, then the $n$-side polygon is reduced to $(n-4)$ decoupled pentagons. Therefore as already discussed, the remainder function in this limit should be reduced to a trivial constant. This constraint provides a way to calculate the periods part by using (3):

$$
A_{\text {periods }}=-\left.\left[\left(A_{\mathrm{BDS}-\text { like }}-A_{\mathrm{BDS}}\right)+A_{\text {extra }}\right]\right|_{\text {all } m_{s} \rightarrow \infty},
$$

up to an constant. Notice that to obtain this relation we have used two important facts. One is that the free energy part simply goes to zero in this limit. The other one is that the periods part has the structure of being quadratic in mass parameters. This is very important so that we do not miss any information in the large mass limit. This property is naturally expected from the definition of the periods part as cycle integrals of the surface, while the contributions which may go zero in the large mass limit are already included in other parts, in particular for the $n=4 K$ case in the $A_{\text {extra }}$ part which contains the information of non-trivial non-compact cycles [23, 27].

Although the idea is very simple and straightforward, in practice there are a few technical issues to consider. One main issue is that the (BDS-BDS-like) part on the right hand side of the formula is expressed in terms of conventional cross ratios. To take the large mass limit, one needs to write them as functions of mass parameters. Generally the relations between cross ratios and mass parameters are not simple, however, in the special limit that we consider these relations turn out to be much simplified. Another complication is that cross ratios are related to $Y$ functions in different phase regions of the spectral parameter. When the phase shift is large, the extra poles terms must be carefully included. This also happens for the $A_{\text {extra }}$ part which involves $T$ function at different phase regions. We mention that there is also a shortcut where there is no need to involve the discussion of pole contributions by simply apply the functional relations, which was explained in section 5.1 .

We study these issues in detail in this paper. The limit for amplitudes up to twelve points are studied. For the known $n \neq 4 K$ cases, we obtain the same results of periods part. This provides a strong consistency check for the validity of our method. We then applie it to calculate the periods part of eight and twelve-point amplitudes for the first time.

The paper is organized as follows. In section 2 we give a brief review of $Y$ system. In section 3. we discuss the relation between cross ratios and $Y$ functions. The pole contribution due to large phase shift is considered in section 4. In section 5 the large mass limit of $Y$ functions and cross ratios is considered. In section 6, we study the amplitudes of $n \neq 4 K$ cases in this limit. The eight and twelve-point cases are considered in section 7 . Some useful formulae and results are collected in three appendices.

\section{Basics of $Y$ system}

In this section we review some basic facts of $Y$ system. We mainly provide the materials that are closely related to the problem studied in this paper, which also set up our conventions. Readers can find more details about the $Y$ system and its derivation for amplitudes at strong coupling in [25, 27]. 
We use the convention that

$$
f^{ \pm}(\theta) \equiv f(\theta \pm i \pi / 4), \quad f^{[l]}(\theta) \equiv f(\theta+i l \pi / 4) .
$$

where $\theta$ is the spectral parameter and can also be written as $\zeta=e^{\theta}$.

We first introduce the $T$ functions which are defined as

$$
\begin{gathered}
T_{0, m}=\left\langle s_{m} s_{m+1} s_{m+2} s_{m+3}\right\rangle^{[-m-1]}, \quad T_{4, m}=\left\langle s_{-2} s_{-1} s_{0} s_{1}\right\rangle^{[-m-1]}, \\
T_{1, m}=\left\langle s_{-2} s_{-1} s_{0} s_{m+1}\right\rangle^{[-m]}, \quad T_{2, m}=\left\langle s_{-1} s_{0} s_{m+1} s_{m+2}\right\rangle^{[-m-1]}, \quad T_{3, m}=\left\langle s_{-1} s_{m} s_{m+1} s_{m+2}\right\rangle^{[-m]},
\end{gathered}
$$

where the contraction is defined as $\left\langle s_{i} s_{i+1} s_{j} s_{j+1}\right\rangle \equiv \epsilon^{\alpha \beta \gamma \delta} s_{i, \alpha} s_{i+1, \beta} s_{j, \gamma} s_{j+1, \delta}$. These $s_{i}$ variables are the smallest solutions of the flat equations which decay fastest to the boundary [25]. Very interestingly they take the same form of the momentum twistor variables, which were first introduced at weak coupling [33] (see also [34]), and are related to ordinary Lorentz variables as

$$
x_{i j}^{2}=\frac{X_{i} \cdot X_{j}}{X_{i}^{+} X_{j}^{+}}, \quad X_{i} \cdot X_{j}=\left\langle s_{i} s_{i+1} s_{j} s_{j+1}\right\rangle, \quad X_{i}^{\alpha \beta} \sim s_{i}^{\alpha} \wedge s_{i+1}^{\beta} .
$$

The $Y$ functions can be constructed from $T$ functions as

$$
Y_{a, m}=\frac{T_{a, m-1} T_{a, m+1}}{T_{a-1, m} T_{a+1, m}} .
$$

They are related to cross ratios as we will see later.

We can impose the normalization conditions

$$
\left\langle s_{i} s_{i+1} s_{i+2} s_{i+3}\right\rangle=1,
$$

with which we have

$$
T_{0, m}=T_{4, m}=T_{a, 0}=1 .
$$

There are also important shifting relations provided by the $Z_{4}$ symmetry of the corresponding $S U(4)$ Hitchin system [25] as

$$
\begin{aligned}
& \left\langle s_{j-1} s_{j} s_{k-1} s_{k}\right\rangle^{[2]}=\left\langle s_{j} s_{j+1} s_{k} s_{k+1}\right\rangle, \\
& \left\langle s_{j-2} s_{j-1} s_{j} s_{k}\right\rangle^{[2]}=\left\langle s_{j} s_{k} s_{k+1} s_{k+2}\right\rangle, \\
& \left\langle s_{j} s_{k-2} s_{k-1} s_{k}\right\rangle^{[2]}=\left\langle s_{j} s_{j+1} s_{j+2} s_{k}\right\rangle .
\end{aligned}
$$

The $T$ functions satisfy the Hirota equation

$$
T_{a, s}^{+} T_{4-a, s}^{-}=T_{4-a, s+1} T_{a, s-1}+T_{a+1, s} T_{a-1, s},
$$

where $a=1,2,3$, and for $n$-point, $s=1,2, \ldots, n-5$. This also implies the following functional relations for $Y$ functions

$$
\begin{aligned}
& \frac{Y_{2, s}^{-} Y_{2, s}^{+}}{Y_{1, s} Y_{3, s}}=\frac{\left(1+Y_{2, s+1}\right)\left(1+Y_{2, s-1}\right)}{\left(1+Y_{1, s}\right)\left(1+Y_{3, s}\right)}, \\
& \frac{Y_{3, s}^{-} Y_{1, s}^{+}}{Y_{2, s}}=\frac{\left(1+Y_{3, s+1}\right)\left(1+Y_{1, s-1}\right)}{1+Y_{2, s}}, \\
& \frac{Y_{1, s}^{-} Y_{3, s}^{+}}{Y_{2, s}}=\frac{\left(1+Y_{1, s+1}\right)\left(1+Y_{3, s-1}\right)}{1+Y_{2, s}} .
\end{aligned}
$$


These functional relations can be written in equivalent but practically more useful integral forms as

$$
\begin{aligned}
& \log Y_{2, s}=-\sqrt{2} m_{s} \cosh \theta-K_{2} \star \alpha_{s}-K_{1} \star \beta_{s}, \\
& \log Y_{1, s}=-m_{s} \cosh \theta-C_{s}-\frac{1}{2} K_{2} \star \beta_{s}-K_{1} \star \alpha_{s}-\frac{1}{2} K_{3} \star \gamma_{s}, \\
& \log Y_{3, s}=-m_{s} \cosh \theta+C_{s}-\frac{1}{2} K_{2} \star \beta_{s}-K_{1} \star \alpha_{s}+\frac{1}{2} K_{3} \star \gamma_{s},
\end{aligned}
$$

where the terms including mass parameters $m_{s}$ and $C_{s}$ are the WKB terms that are related to the boundary conditions. The other functions are defined as

$$
\begin{aligned}
& \alpha_{s} \equiv \log \frac{\left(1+Y_{1, s}\right)\left(1+Y_{3, s}\right)}{\left(1+Y_{2, s-1}\right)\left(1+Y_{2, s+1}\right)}, \quad \gamma_{s} \equiv \log \frac{\left(1+Y_{1, s-1}\right)\left(1+Y_{3, s+1}\right)}{\left(1+Y_{1, s+1}\right)\left(1+Y_{3, s-1}\right)}, \\
& \beta_{s} \equiv \log \frac{\left(1+Y_{2, s}\right)^{2}}{\left(1+Y_{1, s-1}\right)\left(1+Y_{1, s+1}\right)\left(1+Y_{3, s-1}\right)\left(1+Y_{3, s+1}\right)} .
\end{aligned}
$$

and the kernels are

$$
K_{1}(\theta) \equiv \frac{1}{2 \pi} \frac{1}{\cosh \theta}, \quad K_{2}(\theta) \equiv \frac{\sqrt{2}}{\pi} \frac{\cosh \theta}{\cosh 2 \theta}, \quad K_{3}(\theta) \equiv \frac{i}{\pi} \tanh 2 \theta .
$$

Notice the "*" operation can be written explicitly as

$$
K_{i} \star f_{s}=\int_{-\infty}^{+\infty} d \theta^{\prime} K_{i}\left(\theta-\theta^{\prime}\right) f_{s}\left(\theta^{\prime}\right) .
$$

In this form we take the phase $m_{s}$ to be real positive, and the range for the phase of $\zeta$ is $\phi=$ $\operatorname{Im}(\theta) \in(-\pi / 4, \pi / 4)$. In other regions, the above integral equations need to be modified. We will discuss this point in more detail later.

The integral form of $T$ functions can also be given as [27]

$$
\begin{aligned}
& \log T_{2,1}=K_{2} \star \log \left(1+Y_{2,1}\right)+K_{1} \star \log \left(1+Y_{1,1}\right)\left(1+Y_{3,1}\right) \\
& \log T_{1,1}=\frac{1}{2} K_{2} \star \log \left(1+Y_{1,1}\right)\left(1+Y_{3,1}\right)+K_{1} \star \log \left(1+Y_{2,1}\right)+\frac{1}{2} K_{3} \star \log \frac{\left(1+Y_{1,1}\right)}{\left(1+Y_{3,1}\right)}, \\
& \log T_{3,1}=\frac{1}{2} K_{2} \star \log \left(1+Y_{1,1}\right)\left(1+Y_{3,1}\right)+K_{1} \star \log \left(1+Y_{2,1}\right)-\frac{1}{2} K_{3} \star \log \frac{\left(1+Y_{1,1}\right)}{\left(1+Y_{3,1}\right)} .
\end{aligned}
$$

All other $T$ functions can be obtained by using these three $T$ functions and $Y$ functions. For example, by using the relation $T_{2,2}=T_{1,1} T_{3,1} Y_{2,1}$, we can obtain $T_{2,2}$ as

$$
\log T_{2,2}=-\sqrt{2} m_{1} \cosh (\theta)+K_{2} \star \log \left(1+Y_{2,2}\right)+K_{1} \star \log \left(1+Y_{1,2}\right)\left(1+Y_{3,2}\right) .
$$

We emphasize that a special normalization of $T$ functions is chosen, for example one can notice that there is no WKB term in $T_{2,1}$. This freedom of choosing normalization comes from the gauge redundancy of the Hirota equation for $T$ functions [25]. But the full amplitudes are of course independent of such choice. Since our method is based on the consistency condition of the full amplitudes, we do not need to worry about this issue here. 


\section{Cross ratios and $Y$ functions}

As mentioned before, the amplitudes at strong coupling involve parameters $m_{s}$, which are in general related to cross ratios though complicated integral equations of $Y$ functions. To study the collinear limit of amplitudes, we first need to study the relation between cross ratios and $Y$ functions. We will also see that one can obtain a one parameter family of cross ratios which will provide further restriction on the remainder functions.

\subsection{From $Y$ functions to cross ratios}

The traditional cross ratios which involve consecutive cusps can be defined as

$$
u_{i j} \equiv \frac{x_{i, j+1}^{2} x_{i+1, j}^{2}}{x_{i j}^{2} x_{i+1, j+1}^{2}}=\frac{\left\langle s_{i} s_{i+1} s_{j+1} s_{j+2}\right\rangle\left\langle s_{i+1} s_{i+2} s_{j} s_{j+1}\right\rangle}{\left\langle s_{i} s_{i+1} s_{j} s_{j+1}\right\rangle\left\langle s_{i+1} s_{i+2} s_{j+1} s_{j+2}\right\rangle},
$$

where we have written the cross ratios in terms of the small solutions by using (77). A generic cross ratio involving non-consecutive cusps can be constructed by multiplying together consecutive ones, for example

$$
\frac{x_{i, j+2}^{2} x_{i+2, j}^{2}}{x_{i j}^{2} x_{i+2, j+2}^{2}}=u_{i j} u_{i, j+1} u_{i+1, j} u_{i+1, j+1} .
$$

The cross ratios are not all independent. For $n$-point where $n>5$, there are only $3(n-5)$ independent cross ratios, due to the constraints of on-shell condition and Gram determinant constraints [4, 19].

The consecutive-cusp cross ratios can be constructed via $Y_{2, m}$ by using (66)-(8) as

$$
\frac{Y_{2, m}}{1+Y_{2, m}}=\frac{\left\langle s_{-1} s_{0} s_{m+2} s_{m+3}\right\rangle^{[-m-2]}\left\langle s_{-1} s_{0} s_{m} s_{m+1}\right\rangle^{[-m]}}{\left\langle s_{-1} s_{0} s_{m+1} s_{m+2}\right\rangle^{[-m-2]}\left\langle s_{-1} s_{0} s_{m+1} s_{m+2}\right\rangle^{[-m]}} .
$$

When $m=2 k-2$, we have

$$
\frac{Y_{2,2 k-2}}{1+Y_{2,2 k-2}}=\frac{\left\langle s_{-k-1} s_{-k} s_{k} s_{k+1}\right\rangle\left\langle s_{-k} s_{-k+1} s_{k-1} s_{k}\right\rangle}{\left\langle s_{-k-1} s_{-k} s_{k-1} s_{k}\right\rangle\left\langle s_{-k} s_{-k+1} s_{k} s_{k+1}\right\rangle}=\frac{x_{-k-1, k}^{2} x_{-k, k-1}^{2}}{x_{-k-1, k-1}^{2} x_{-k, k}^{2}} .
$$

When $m=2 k-1$, we have

$$
\left(\frac{Y_{2,2 k-1}}{1+Y_{2,2 k-1}}\right)^{+}=\frac{\left\langle s_{-k-1} s_{-k} s_{k+1} s_{k+2}\right\rangle\left\langle s_{-k} s_{-k+1} s_{k} s_{k+1}\right\rangle}{\left\langle s_{-k-1} s_{-k} s_{k} s_{k+1}\right\rangle\left\langle s_{-k} s_{-k+1} s_{k+1} s_{k+2}\right\rangle}=\frac{x_{-k-1, k+1}^{2} x_{-k, k}^{2}}{x_{-k-1, k}^{2} x_{-k, k+1}^{2}} .
$$

Using the shifting relation (11), we can write all consecutive cross ratios in terms of $Y$ functions.

As an explicit example, for the simplest six-point case, there are three independent cross ratios

$$
u_{14}=\frac{x_{15}^{2} x_{24}^{2}}{x_{14}^{2} x_{25}^{2}}, \quad u_{25}=\frac{x_{26}^{2} x_{35}^{2}}{x_{25}^{2} x_{36}^{2}} . \quad u_{36}=\frac{x_{13}^{2} x_{46}^{2}}{x_{36}^{2} x_{14}^{2}} .
$$

We can express them in terms of $Y$ functions as

$$
u_{36}=\frac{Y_{2,1}^{-}}{1+Y_{2,1}^{-}}, \quad u_{14}=\frac{Y_{2,1}^{+}}{1+Y_{2,1}^{+}}, \quad u_{25}=\frac{Y_{2,1}^{[3]}}{1+Y_{2,1}^{[3]}} .
$$

The relations between $Y$ functions and the cross ratios that are commonly used in weak coupling calculation for higher-point cases are given explicitly in appendix A. 


\subsection{One parameter family of cross ratios}

$Y$ functions (and therefore also cross ratios) are functions of spectral parameter. The physical cross ratios of the original problem can be recovered by taking $\zeta=e^{\theta}=1$. A very interesting fact is that one can actually obtain a one parameter family of physical cross ratios by taking $\zeta$ to be a pure phase, or equivalently $\theta$ to be a pure imaginary number $i \phi[25]$. We can write this one family of cross ratios by including the $\phi$-dependence as

$$
u_{36}(i \phi)=\frac{Y_{2,1}^{-}(i \phi)}{1+Y_{2,1}^{-}(i \phi)}, \quad u_{14}(i \phi)=\frac{Y_{2,1}^{+}(i \phi)}{1+Y_{2,1}^{+}(i \phi)}, \quad u_{25}(i \phi)=\frac{Y_{2,1}^{[3]}(i \phi)}{1+Y_{2,1}^{[3]}(i \phi)} .
$$

An important fact is that the free energy and periods parts are independent of the phase $\phi$, and therefore take same values for the whole family of cross ratios. This is due to the integrability of the world-sheet theory 25] 1 . This invariance provides a further constraint for the amplitudes, which is important for the limit that we consider in this paper. Although $A_{\text {free }}$ and $A_{\text {periods }}$ are independent of the phase, the other parts of remainder function do depend. Therefore, the remainder function also depends on $\phi$ as

$$
R(\phi)=\left(A_{\mathrm{BDS}-\text { like }}-A_{\mathrm{BDS}}\right)(\phi)+A_{\text {extra }}(\phi)+A_{\text {periods }}+A_{\text {free }} .
$$

On the other hand, the limit we consider which gives rise to (4) is still true, therefore we have that

$$
A_{\text {periods }}=-\left.\left[\left(A_{\mathrm{BDS}-\mathrm{like}}-A_{\mathrm{BDS}}\right)(\phi)+A_{\mathrm{extra}}(\phi)\right]\right|_{\text {all } m_{s} \rightarrow \infty} .
$$

This provides a stronger version of (44). Since each part on the right hand side depends on the phase separately, there must be non-trivial cancellation, so that the finally result is independent of $\phi$ as required by the equality. We will see that this is indeed true. This restriction provides another important consistency check for our method.

\section{Phase shift and pole terms}

To obtain cross ratio, we need to consider $Y$ functions with large phase shift

$$
Y_{2, s}^{[r]}(\theta)=Y_{2, s}(\theta+i r \pi / 4) .
$$

This will require a modification of the integral equations. The reason is that residue contributions should be included in the integral equations when we cross the pole lines of kernel functions. Mathematically, this is similar to the wall crossing phenomenon in the study of supersymmetric field theory [30, 31].

In general there are two cases that one needs to consider pole contribution. One is the argument $\theta$ of $Y$ functions is fixed, but the phase of mass parameters changes. The other case is what we have encountered above, where the mass parameters are fixed, but the argument $\theta$ (or more exactly its imaginary part) of $Y$ functions varies. The mathematical reason for both cases is the same, but the physical pictures are different. We are mainly interested in the latter case. We will take

\footnotetext{
${ }^{1}$ It is very interesting to see if there is any similar invariance at weak coupling, which may provide a connection between weak coupling Yangian symmetry and the integrability at strong coupling
} 
the mass parameters as real positive numbers 2 , then the integral equations for $Y$ functions will preserve their original form as long as $\phi=\operatorname{Im}(\theta) \in(-\pi / 4, \pi / 4)$

$$
\begin{aligned}
& \log Y_{2, s}(\theta)=-\sqrt{2} m_{s} \cosh (\theta)-K_{2} \star \alpha_{s}-K_{1} \star \beta_{s}, \\
& \log Y_{1, s}(\theta)=-m_{s} \cosh (\theta)-C_{s}-\frac{1}{2} K_{2} \star \beta_{s}-K_{1} \star \alpha_{s}-\frac{1}{2} K_{3} \star \gamma_{s}, \\
& \log Y_{3, s}(\theta)=-m_{s} \cosh (\theta)+C_{s}-\frac{1}{2} K_{2} \star \beta_{s}-K_{1} \star \alpha_{s}+\frac{1}{2} K_{3} \star \gamma_{s} .
\end{aligned}
$$

Beyond that region, one needs to modify the equations by including extra pole contributions while $\theta$ crosses the lines of $\pm i \pi / 4, \pm i \pi / 2, \cdots$ of $\zeta$ plane. Explicitly, the positions of the poles $\theta_{p}$ of each kernel are

$$
\begin{array}{rlrl}
K_{1} & =\frac{1}{2 \pi} \frac{1}{\cosh \theta}, & \theta_{p} & =i \frac{(1+2 l) \pi}{2}, \\
K_{2}=\frac{\sqrt{2}}{\pi} \frac{\cosh \theta}{\cosh 2 \theta}, & \theta_{p} & =i \frac{(1+2 l) \pi}{4}, \\
K_{3} & =\frac{i}{\pi} \tanh 2 \theta, & \theta_{p} & =i \frac{(1+2 l) \pi}{4},
\end{array}
$$

As an explicit example, we consider $Y_{1, s}(\theta)$ in the case where $\operatorname{Im}(\theta) \in(3 \pi / 4, \pi)$. It crosses three pole lines. While crossing the $\operatorname{line} \operatorname{Im}(\theta)=\pi / 4$, we have pole contributions from kernels $K_{2}$ and $K_{3}$. When crossing the $\operatorname{line} \operatorname{Im}(\theta)=\pi / 2$, we have pole contribution from kernels $K_{1}$. And while crossing the line $\operatorname{Im}(\theta)=3 \pi / 4$, we have pole contributions from kernels $K_{2}$ and $K_{3}$ again. In total, for $Y_{1, s}(\theta)$ with $\operatorname{argument} \operatorname{Im}(\theta) \in(3 \pi / 4, \pi)$ we have

$$
\begin{aligned}
\log Y_{1, s}(\theta) & =-m_{s} \cosh (\theta)-C_{s}-\frac{1}{2} K_{2} \star \beta_{s}-K_{1} \star \alpha_{s}-\frac{1}{2} K_{3} \star \gamma_{s} \\
& -\frac{1}{2} \beta_{s}(\theta-i \pi / 4)+\frac{1}{2} \gamma_{s}(\theta-i \pi / 4)-\alpha_{s}(\theta-i \pi / 2)-\frac{1}{2} \beta_{s}(\theta-i 3 \pi / 4)+\frac{1}{2} \gamma_{s}(\theta-i 3 \pi / 4) .
\end{aligned}
$$

Notice that one should also be careful about the signs of residues.

It is convenient to use another notation when we consider cross ratios. For example, for six-point we want to calculate

$$
Y_{2,1}^{-}(i \phi)=Y_{2,1}(i \phi-i \pi / 4), \quad Y_{2,1}^{+}(i \phi)=Y_{2,1}(i \phi+i \pi / 4), \quad Y_{2,1}^{[3]}(i \phi)=Y_{2,1}(i \phi+i 3 \pi / 4) .
$$

We choose $\phi \in(0, \pi / 4)$. We can see that there is no pole contribution for $Y_{2,1}^{-}(i \phi)$, but there are pole contributions for the other two functions. Similar to the example above, we can obtain that

$$
\begin{aligned}
& \log Y_{2,1}^{-}(i \phi)=-\sqrt{2} m_{1} \cos (\phi-\pi / 4)-K_{2} \star \alpha_{1}-K_{1} \star \beta_{1}, \\
& \log Y_{2,1}^{+}(i \phi)=-\sqrt{2} m_{1} \cos (\phi+\pi / 4)-K_{2} \star \alpha_{1}-K_{1} \star \beta_{1}-\alpha_{1}(i \phi), \\
& \log Y_{2,1}^{[3]}(i \phi)=-\sqrt{2} m_{1} \cos (\phi+3 \pi / 4)-K_{2} \star \alpha_{1}-K_{1} \star \beta_{1}-\alpha_{1}^{[2]}(i \phi)-\beta_{1}^{[1]}(i \phi)-\alpha_{1}(i \phi) .
\end{aligned}
$$

We can then define the cross ratios as in $(35)]^{3}$. In appendix $\mathrm{B}$, we provide equations for more complicated cases which we will encounter when we calculate higher points amplitudes. Since we

\footnotetext{
${ }^{2}$ Notice that for simplicity, we take $m_{s}$ to be real positive number. One can of course include a phase $\varphi_{s}$ for each $m_{s}$. Then one may need to modify the equations also according to the range of $\varphi_{s}$ [25. The discussion will be more involved but straightforward.

${ }^{3}$ One may notice that the integral equations for cross ratios looks a little different from that in [24] (see also [35]). They are equivalent but written in different presentations, and are related by the functional relations. One can also explicitly solve the the integral equations numerically and find they give the same cross ratios.
} 
need to calculate $T$ functions when there is $A_{\text {extra }}$ part, the equations for $T_{2,1}, T_{2,2}$ with large phase shift are also given.

We should mention that these integral equations are correct no matter whether we take the large mass limit or not. Therefore they can be used for more general studies. Numerically they can be solved very efficiently at least when the number of gluons is not very large. There are two very useful checks for the equations and results. One is that in the small $m_{s}$ limit, the $Y$ functions should converge to the same value which is independent of $\phi$. This corresponds to the regular polygon limit, where all cross ratios take the same value. The other more non-trivial check is the continuous condition that, $Y$ functions themselves must be continuous functions for all phase, despite that the expressions of $Y$ functions in different regions of phase look different due to extra pole terms. In other words, one needs to check that there is no discontinuity when the pole lines are crossed. We have done these checks for the equations we give.

\section{$5 \quad$ Large mass limit of $Y$ functions}

When all $m_{s}$ are taken to be infinitely large, the relations between cross ratios and mass parameters become much simpler. The main reason is that the complicated integral terms always vanish in the limit, as

$$
K \star \log \left(1+Y_{a, s}\right)=\int_{-\infty}^{+\infty} d \theta K \log \left(1+e^{-m_{s} \cosh \theta+\cdots}\right) \rightarrow 0
$$

Notice that for simplicity we take $m_{s}$ to be real positive. Therefore, the $Y$ functions are dominated by the WKB terms in the limit. The main complication comes from that the pole terms may also have contribution in the limit.

We consider six-point case explicitly. We first consider $u_{36}$ in (34). In the large $m_{1}$ limit, we can neglect the integral terms in (47) and have

$$
\log Y_{2,1}^{-}(i \phi) \rightarrow-\sqrt{2} m_{1} \cos (\phi-\pi / 4)
$$

Then we have

$$
u_{36}(i \phi)=\frac{Y_{2,1}^{[-1]}(i \phi)}{1+Y_{2,1}^{[-1]}(i \phi)} \rightarrow \frac{e^{-\sqrt{2} m_{1} \cos (\phi-\pi / 4)}}{1+e^{-\sqrt{2} m_{1} \cos (\phi-\pi / 4)}} \rightarrow e^{-\sqrt{2} m_{1} \cos (\phi-\pi / 4)}
$$

Notice that the sign of $\log Y$ in the limit makes the results totally different. Here it is negative. If it is positive, then $Y \rightarrow+\infty$ and the corresponding cross ratio will simply be one in the limit.

For $u_{14}$ we need to consider $Y_{2,1}^{+}(i \phi)$, where there is a pole term

$$
\begin{aligned}
\alpha_{1}(i \phi) & =\log \left(1+Y_{1,1}(i \phi)\right)+\log \left(1+Y_{3,1}(i \phi)\right) \\
& \rightarrow \log \left(1+e^{-m_{1} \cos (\phi)+C_{1}}\right)+\log \left(1+e^{-m_{1} \cos (\phi)-C_{1}}\right) .
\end{aligned}
$$

In the second line we take the limit and neglect the integral terms in $Y_{1,1}$ and $Y_{3,1}$. Comparing to WKB terms in $Y_{2,1}^{+}$, this pole contribution is exponentially suppressed and can be neglected, therefore we have

$$
\log Y_{2,1}^{+}(i \phi) \rightarrow-\sqrt{2} m_{1} \cos (\phi+\pi / 4)
$$


and the cross ratio is

$$
u_{14}(i \phi) \rightarrow e^{-\sqrt{2} m_{1} \cos (\phi+\pi / 4)}
$$

Similarly we consider $u_{25}$. We can see from (21) that the pole terms are always a summation of functions in the form of $\log (1+Y)$, therefore when $Y \rightarrow e^{-\infty}$ the contribution can always be neglected. But when $Y \rightarrow e^{+\infty}$ there is non-trivial contribution, which turns out to be the case for the pole term $\alpha_{1}^{[2]}(i \phi)$ in $Y_{2,1}^{[3]}(i \phi)$. We have

$$
\begin{aligned}
\alpha_{1}^{[2]}(i \phi) & =\log \left(1+Y_{1,1}^{[2]}(i \phi)\right)+\log \left(1+Y_{3,1}^{[2]}(i \phi)\right) \\
& \rightarrow \log \left(1+e^{-m_{1} \cos (\phi+\pi / 2)+C_{1}}\right)+\log \left(1+e^{-m_{1} \cos (\phi+\pi / 2)-C_{1}}\right) \\
& \rightarrow\left(m_{1} \sin (\phi)+C_{1}\right)+\left(m_{1} \sin (\phi)-C_{1}\right) \\
& \rightarrow 2 m_{1} \sin (\phi)
\end{aligned}
$$

Notice the parameters $C_{s}$ always cancel with each other in the limit, since $Y_{1, s}$ and $Y_{3, s}$ always appear in pairs. The other pole terms in (49) are exponential suppressed, therefore in total we have

$$
\log Y_{2,1}^{[3]}(i \phi) \rightarrow \sqrt{2} m_{1} \sin (\phi+\pi / 4)-2 m_{1} \sin (\phi)
$$

We can see that $Y_{2,1}^{[3]} \rightarrow+\infty$ for $\phi \in(0, \pi / 4)$, therefore $u_{25}$ is simply one in the limit.

To summarize we have the cross ratios in the large mass limit as

$$
\left\{u_{36}(i \phi), u_{14}(i \phi), u_{25}(i \phi)\right\} \rightarrow\left\{e^{-\sqrt{2} m_{1} \cos (\phi-\pi / 4)}, e^{-\sqrt{2} m_{1} \cos (\phi+\pi / 4)}, 1\right\} .
$$

The results including the cross ratios of several higher-point cases and $T$ functions will be given in the following sections. They can be derived in the same way.

\subsection{Another way bypassing the pole contributions}

There is another way to obtain the large mass limit of the cross ratios in which one does not need to consider the pole contributions. The idea is that one can always use the functional relations (15)-(17) to bring $Y_{a, s}^{[r]}$ to a combination of $Y_{a, s}^{[r-1]}$ and $Y_{a, s}^{[r-2]}$, or to a combination of $Y_{a, s}^{[r+1]}$ and $Y_{a, s}^{[r+2]}$. Thus it is always possible to express all cross ratios in terms of only $Y_{a, s}^{[0]}$ and $Y_{a, s}^{[-1]}$ for which the phases are in the region $\phi \in(-\pi / 4, \pi / 4)$. Then one can simply apply the relation

$$
\begin{aligned}
& \log Y_{2, s}(i \phi) \simeq-\sqrt{2} m_{s} \cosh (\phi) \\
& \log Y_{1, s}(i \phi) \simeq-m_{s} \cosh (\phi)-C_{s} \\
& \log Y_{3, s}(i \phi) \simeq-m_{s} \cosh (\phi)+C_{s}
\end{aligned}
$$

to obtain the large mass limit of the cross ratios.

Using Hirota equation (14), one can similarly calculate the $T$ functions which appear in $A_{\text {extra }}$ part in the same way.

\footnotetext{
${ }^{4}$ We would like to thank the anonymous referee for this suggestion.
} 


\section{Consistency check for the known results}

We first apply out method to the $n \neq 4 K$ case, where there is no $A_{\text {extra }}$ part. The periods can be calculated as

$$
A_{\text {periods }}=-\left.\left[\left(A_{\mathrm{BDS}-\mathrm{like}}-A_{\mathrm{BDS}}\right)(\phi)\right]\right|_{\text {all } m_{s} \rightarrow \infty} .
$$

We will reproduce all known results for up to eleven points. We summarize the results of amplitudes which may be used here in appendix $\mathrm{C}$.

\subsection{Six-point}

The results of periods part and the difference between BDS-like and BDS part are known [24]

$$
\begin{aligned}
& A_{\text {periods }}^{n=6}=\frac{\left|m_{1}\right|^{2}}{4}, \\
& A_{\mathrm{BDS}-\mathrm{like}}^{n=6}-A_{\mathrm{BDS}}^{n=6}=-\sum_{i=1}^{3}\left(\frac{1}{8} \log ^{2} u_{i, i+3}+\frac{1}{4} L i_{2}\left(1-u_{i, i+3}\right)\right) .
\end{aligned}
$$

The cross ratios in the large mass limit have been obtained above

$$
\left\{u_{36}(i \phi), u_{14}(i \phi), u_{25}(i \phi)\right\} \rightarrow\left\{e^{-\sqrt{2} m_{1} \cos (\phi-\pi / 4)}, e^{-\sqrt{2} m_{1} \cos (\phi+\pi / 4)}, 1\right\} .
$$

Applying this limit in (62) we obtain that

$$
\begin{aligned}
\left(A_{\mathrm{BDS}-\text { like }}^{n=6}-A_{\mathrm{BDS}}^{n=6}\right)(\phi) & \rightarrow-\left(\frac{1}{8} 2 m_{1}^{2}\left[\cos ^{2}(\phi-\pi / 4)+\cos ^{2}(\phi+\pi / 4)\right]+\frac{1}{4} L i_{2}(1)\right) \\
& =-\frac{1}{4} m_{1}^{2}+\text { constant }
\end{aligned}
$$

which indeed cancels with $A_{\text {periods }}$ up to a constant. Although we have choose $m_{s}$ to be real positive number for simplicity, this is enough to reproduce the more general expression (61) by knowing that the periods part is real. One can of course also do an honest calculation, for example consider to include a small phase factor for $m_{s}$ (so that the equations do not change) and find that the result is indeed correct.

We can see that in the limit, although cross ratios still depends on the phase $\phi$, the difference between the BDS-like and BDS part is independent of $\phi$. We emphasize that this independence is not true in general, it only happens in the limit, which is required to cancel the periods part. As we will see in the $n=4 K$ case, such cancellation will become more non-trivial due to the existence of extra part.

\subsection{Seven-point}

We have the result

$$
\begin{aligned}
& A_{\text {periods }}^{n=7}=\frac{\left|m_{1}\right|^{2}+\left|m_{2}\right|^{2}}{2}+\frac{m_{1} \bar{m}_{2}+\bar{m}_{1} m_{2}}{2 \sqrt{2}}, \\
& A_{\mathrm{BDS}-\text { like }}^{n=7}-A_{\mathrm{BDS}}^{n=7}=-\frac{1}{4} \sum_{i=1}^{7}\left(\log ^{2} u_{i, i+3}+L i_{2}\left(1-u_{i, i+3}\right)-\frac{1}{2} \log u_{i, i+3} \log \frac{u_{i+2, i+5} u_{i+1, i+5}}{u_{i+3, i+6} u_{i, i+4}}\right) .
\end{aligned}
$$


The cross ratios in the large mass limit can be obtained as

$$
\begin{aligned}
& \left\{u_{14}(i \phi), u_{25}(i \phi), u_{36}(i \phi), u_{47}(i \phi), u_{15}(i \phi), u_{26}(i \phi), u_{37}(i \phi)\right\} \rightarrow \\
& \quad\left\{e^{-\sqrt{2} m_{2} \cos (\phi)}, 1,1, e^{-\sqrt{2} m_{1} \cos (\phi-\pi / 4)}, e^{-\sqrt{2} m_{1} \cos (\phi+\pi / 4)}, 1, e^{-\sqrt{2} m_{2} \sin (\phi)}\right\} .
\end{aligned}
$$

Applying this limit in (66) we find that

$$
\left(A_{\mathrm{BDS}-\text { like }}^{n=7}-A_{\mathrm{BDS}}^{n=7}\right)(\phi) \rightarrow-\frac{m_{1}^{2}+m_{2}^{2}}{2}-\frac{m_{1} m_{2}}{\sqrt{2}}+\text { constant },
$$

which is independent of the $\phi$, and consistent with the known result of periods part (65).

\subsection{Nine-point}

We have the results that

$$
\begin{aligned}
& A_{\text {periods }}^{n=9}=-\frac{1}{2}\left(\left|m_{2}\right|^{2}+\left|m_{3}\right|^{2}+m_{1} \bar{m}_{3}+m_{3} \bar{m}_{1}+m_{2} \bar{m}_{4}+m_{4} \bar{m}_{2}\right) \\
&-\frac{\sqrt{2}}{4}\left(m_{1} \bar{m}_{2}+m_{2} \bar{m}_{1}+m_{1} \bar{m}_{4}+m_{4} \bar{m}_{1}+m_{3} \bar{m}_{4}+m_{4} \bar{m}_{3}+2 m_{2} \bar{m}_{3}+2 m_{3} \bar{m}_{2}\right) \\
& A_{\mathrm{BDS}-\text { like }}^{n=9}-A_{\mathrm{BDS}}^{n=9}= \frac{1}{4} \sum_{i=1}^{9}\left[\log u_{i, i+3} \log \left(\frac{u_{i, i+4} u_{i+4, i+7}}{u_{i+1, i+5} u_{i+2, i+5} u_{i+3, i+6} u_{i+2, i+6}^{2}}\right)\right. \\
&\left.\quad+\log u_{i, i+4} \log \left(\frac{u_{i, i+4} u_{i+1, i+4} u_{i+4, i+8}^{2} u_{i+5, i+8}^{2}}{u_{i+2, i+5} u_{i+2, i+6}^{3} u_{i+3, i+6}^{2} u_{i+3, i+7}^{2}}\right)-L i_{2}\left(1-u_{i, i+3}\right)-L i_{2}\left(1-u_{i, i+4}\right)\right] .
\end{aligned}
$$

The cross ratios in the large mass limit can be obtained as

$$
\begin{gathered}
\left\{u_{14}(i \phi), u_{25}(i \phi), u_{36}(i \phi), u_{47}(i \phi), u_{58}(i \phi), u_{69}(i \phi), u_{17}(i \phi), u_{28}(i \phi), u_{39}(i \phi)\right\} \rightarrow \\
\left\{e^{-\sqrt{2} m_{4} \sin (\phi)}, e^{-\sqrt{2} m_{4} \cos (\phi)}, 1,1,1, e^{-\sqrt{2} m_{1} \cos (\phi-\pi / 4)}, e^{-\sqrt{2} m_{1} \cos (\phi+\pi / 4)}, 1,1\right\} \\
\left\{u_{15}(i \phi), u_{26}(i \phi), u_{37}(i \phi), u_{48}(i \phi), u_{59}(i \phi), u_{16}(i \phi), u_{27}(i \phi), u_{38}(i \phi), u_{49}(i \phi)\right\} \rightarrow \\
\left\{e^{-\sqrt{2} m_{3} \cos (\phi-\pi / 4)}, e^{-\sqrt{2} m_{3} \cos (\phi+\pi / 4)}, 1,1, e^{-\sqrt{2} m_{2} \sin (\phi)}, e^{-\sqrt{2} m_{2} \cos (\phi)}, 1,1,1\right\} .
\end{gathered}
$$

One can find in this limit that

$$
\begin{aligned}
& \left(A_{\mathrm{BDS}-\text { like }}^{n=9}-A_{\mathrm{BDS}}^{n=9}\right)(\phi) \rightarrow \\
& \quad \frac{1}{2}\left(m_{2}^{2}+m_{3}^{2}+2 m_{1} m_{3}+2 m_{2} m_{4}\right)+\frac{\sqrt{2}}{2}\left(m_{1} m_{2}+m_{1} m_{4}+m_{3} m_{4}+2 m_{2} m_{3}\right)+\text { constant }
\end{aligned}
$$

which is independent of the $\phi$, and consistent with the result of periods part (69).

We also calculate the ten and eleven-point cases and also reproduce the periods part given in [25]. Since these checks are very non-trivial, it provides a strong check for the validity of our method. We will consider $n=4 K$ cases in next section.

\section{$7 \quad$ Fix the periods part for $n=4 K$ cases}

For $n=4 K$ case, we can calculate the periods parts by using

$$
A_{\text {periods }}=-\left.\left[\left(A_{\mathrm{BDS}-\mathrm{like}}-A_{\mathrm{BDS}}\right)(\phi)+A_{\text {extra }}(\phi)\right]\right|_{\text {all } m_{s} \rightarrow \infty} .
$$


For eight point, the cutoff part of the amplitudes was calculated in [27]. We can obtain that

$$
\begin{aligned}
A_{\mathrm{BDS}-\text { like }}^{n=8}-A_{\mathrm{BDS}}^{n=8}= & -\frac{1}{8} \log ^{2}\left(\frac{u_{14} u_{25} u_{58} u_{16}}{u_{36} u_{47} u_{27}}\right)-\frac{1}{4} \log \left(u_{15} u_{16} u_{25} u_{26}\right) \log \left(u_{26} u_{27} u_{36} u_{37}\right) \\
& -\frac{1}{2} \log \left(\frac{u_{15}}{u_{37}}\right) \log \left(\frac{u_{14} u_{25} u_{58} u_{16} u_{15}}{u_{36} u_{47} u_{27} u_{37}}\right)-\frac{1}{8}\left(\Delta_{x}-\Delta_{y}\right)^{2} \\
& +\frac{1}{4} \Delta_{x} \log \left(\frac{u_{14} u_{15}^{2} u_{16} u_{25} u_{58}}{u_{27} u_{36} u_{37}^{2} u_{47}}\right)+\frac{1}{4} \Delta_{y} \log \left(\frac{u_{26} u_{27} u_{36} u_{37}^{2} u_{47}}{u_{14} u_{15} u_{58}}\right) \\
& -\frac{1}{8} \sum_{i=1}^{8} \log ^{2} u_{i, i+3}-\frac{1}{8} \sum_{i=1}^{8}\left[2 L i_{2}\left(1-u_{i, i+3}\right)+L i_{2}\left(1-u_{i, i+4}\right)\right], \\
A_{\text {extra }}^{n=8}= & -\frac{1}{4}\left(\Delta_{x} \log T_{2,2}^{[-3]}-\Delta_{y} \log T_{2,2}^{[-5]}\right),
\end{aligned}
$$

where $\Delta_{x, y}$ are given in (129). We can see the difference between BDS-like and BDS parts is indeed only a function of cross ratios. The cross ratios in the large mass limit are

$$
\begin{aligned}
& \left\{u_{14}(i \phi), u_{25}(i \phi), u_{36}(i \phi), u_{47}(i \phi), u_{58}(i \phi), u_{16}(i \phi), u_{27}(i \phi), u_{38}(i \phi)\right\} \rightarrow \\
& \quad\left\{e^{-\sqrt{2} m_{3} \cos (\phi-\pi / 4)}, e^{-\sqrt{2} m_{3} \cos (\phi+\pi / 4)}, 1,1, e^{-\sqrt{2} m_{1} \cos (\phi-\pi / 4)}, e^{-\sqrt{2} m_{1} \cos (\phi+\pi / 4)}, 1,1\right\}, \\
& \left\{u_{15}(i \phi), u_{26}(i \phi), u_{37}(i \phi), u_{48}(i \phi)\right\} \rightarrow\left\{e^{-\sqrt{2} m_{2} \cos (\phi)}, 1,1, e^{-\sqrt{2} m_{2} \sin (\phi)}\right\} .
\end{aligned}
$$

$\Delta_{x, y}$ are two special cross ratios that are related to the monodromy [27], and can be given exactly as

$$
\begin{aligned}
& \Delta_{x}(\phi)=-\sqrt{2}\left(m_{1}+\sqrt{2} m_{2}+m_{3}\right) \cos (\phi+\pi / 4), \\
& \Delta_{y}(\phi)=-\sqrt{2}\left(m_{1}+\sqrt{2} m_{2}+m_{3}\right) \sin (\phi+\pi / 4) .
\end{aligned}
$$

We also have extra part which involves $T$ functions. In the same way as that for $Y$ function, we can obtain that

$$
\begin{aligned}
& \log T_{2,2}^{[-3]}(\phi) \rightarrow \sqrt{2} m_{1} \cos (\phi+\pi / 4) \\
& \log T_{2,2}^{[-5]}(\phi) \rightarrow\left(2 m_{1}+\sqrt{2} m_{2}+m_{3}\right) \cos (\phi)-m_{3} \sin (\phi) .
\end{aligned}
$$

By substituting (77)-(79) into (75) and (76), we obtain

$$
\begin{aligned}
\left(A_{\mathrm{BDS}-\text { like }}^{n=8}-A_{\mathrm{BDS}}^{n=8}\right)(\phi) \rightarrow & -\frac{\sqrt{2} m_{2}}{8}\left(m_{1}+\sqrt{2} m_{2}+m_{3}\right)(1+\sin (2 \phi)-\cos (2 \phi)) \\
& -\frac{1}{4}\left(m_{1}+\sqrt{2} m_{2}+m_{3}\right)^{2} \cos (2 \phi)-\frac{m_{1}}{2}\left(m_{1}+\sqrt{2} m_{2}+m_{3}\right) \\
& -\frac{m_{2}^{2}+m_{3}^{2}+\sqrt{2} m_{2} m_{3}}{2}+\frac{1}{4}\left(m_{1}+\sqrt{2} m_{2}+m_{3}\right)^{2}, \\
A_{\text {extra }}^{n=8}(\phi) \rightarrow & \frac{\sqrt{2} m_{2}}{8}\left(m_{1}+\sqrt{2} m_{2}+m_{3}\right)(1+\sin (2 \phi)-\cos (2 \phi)) \\
& +\frac{1}{4}\left(m_{1}+\sqrt{2} m_{2}+m_{3}\right)^{2} \cos (2 \phi)+\frac{m_{1}}{2}\left(m_{1}+\sqrt{2} m_{2}+m_{3}\right) .
\end{aligned}
$$

We can see that $\left(A_{\mathrm{BDS}-\text { like }}^{n=8}-A_{\mathrm{BDS}}^{n=8}\right)$ and $A_{\text {extra }}$ both depend on $\phi$ in a non-trivial way. However, gratifyingly, the summation of them is independent of $\phi$, and we have

$$
\left(A_{\mathrm{BDS}-\text { like }}^{n=8}-A_{\mathrm{BDS}}^{n=8}\right)(\phi)+A_{\mathrm{extra}}^{n=8}(\phi) \rightarrow-\frac{m_{2}^{2}+m_{3}^{2}+\sqrt{2} m_{2} m_{3}}{2}+\frac{1}{4}\left(m_{1}+\sqrt{2} m_{2}+m_{3}\right)^{2} .
$$


This non-trivial cancellation for the $\phi$-dependent terms is a strong check for the result. The periods part is then given by (74) as

$$
A_{\text {periods }}^{n=8}=\frac{\left|m_{2}\right|^{2}+\left|m_{3}\right|^{2}}{2}+\frac{m_{2} \bar{m}_{3}+\bar{m}_{2} m_{3}}{2 \sqrt{2}}-\frac{1}{4}\left|m_{1}+\sqrt{2} m_{2}+m_{3}\right|^{2},
$$

where we have written it for general complex $m_{s}$ by using the real condition. Comparing with the periods part conjectured in [27], we can see we need to add a new term

$$
-\frac{1}{4}\left|m_{1}+\sqrt{2} m_{2}+m_{3}\right|^{2}=-\frac{1}{4}\left|w_{0}\right|^{2}
$$

where $w_{0} \equiv m_{1}+\sqrt{2} m_{2}+m_{3}$ is the monodromy [26, 27]. Structurally, one may also write the periods of eight-point as

$$
A_{\text {periods }}^{n=8}=\left.A_{\text {periods }}^{n=7}\right|_{m_{i} \rightarrow m_{i+1}}-\frac{1}{4}\left|w_{0}\right|^{2} .
$$

We also calculate the twelve-points, and as well find the non-trivial cancellation for $\phi$ dependent terms. The periods part also shows interesting structure as

$$
A_{\text {periods }}^{n=12}=\left.A_{\text {periods }}^{n=11}\right|_{m_{i} \rightarrow m_{i+1}}+\left.A_{\text {periods }}^{n=10}\right|_{m_{i} \rightarrow m_{i+1}}-\frac{1}{4}\left|w_{0}^{n=12}\right|^{2}
$$

where each term on the right hand side may be found in [25]. This also shows that the conjectured result in [27] is not a full answer, which includes only the first term.

\section{Conclusion}

In this paper, we study a special collinear limit of the amplitudes at strong coupling which in the $Y$ system corresponds to a limit that all mass parameters are taken to be very large. This limit imposes a strong restriction on the amplitudes by which the periods parts can be uniquely fixed by the already known other parts of the amplitudes. This is particularly important for the case where the number of gluons is a multiple of four, since the results of periods part have not been calculated before. We do the calculation for amplitudes up to twelve points. For the $n \neq 4 K$ cases, we all reproduce the known results, which provides a strong consistency check for the method. We calculate the periods part for eight and twelve points for the first time. The non-trivial cancellation of the $\phi$-dependent terms shows that the calculations are consistent. We also study some technical issues in detail which are involved in the calculation, such as the relation between traditional cross ratios and $Y$ function and their large mass limit. The pole contributions due to large phase shift of the spectral parameter are also discussed and the explicit integral equations of $Y$ and $T$ functions are given. These relations can be applied not only in the large

mass limit considered in this paper, but also in more general study of the amplitudes.

\section{Acknowledgements}

The author has benefited from the discussion with L. Alday, A. Brandhuber, E. Buchbinder, A. Sever, and G. Travaglini. He is grateful to Brandhuber and Travaglini for the suggestion and encouragement. He would like to thank the invitation of Imperial college where this work was presented. The author would also like to thank the anonymous referee for his or her careful reading of the manuscript, and the valuable comments and suggestions for the improvements. This work is supported by STFC. 


\section{A Explicit relations between cross ratios from $Y$ functions}

We write cross ratios that are commonly used in weak coupling calculations in terms of $Y$ functions explicitly.

\section{A.1 Six-point}

For six-point case, we have

$$
u_{14} \equiv \frac{x_{15}^{2} x_{24}^{2}}{x_{14}^{2} x_{25}^{2}}, \quad u_{25} \equiv \frac{x_{26}^{2} x_{35}^{2}}{x_{25}^{2} x_{36}^{2}}, \quad u_{36} \equiv \frac{x_{13}^{2} x_{46}^{2}}{x_{36}^{2} x_{14}^{2}} .
$$

Using $Y$ functions we obtain a one parameter family of cross ratios

$$
u_{36}=\frac{Y_{2,1}^{[-1]}}{1+Y_{2,1}^{[-1]}}, \quad u_{14}=\frac{Y_{2,1}^{+}}{1+Y_{2,1}^{+}}, \quad u_{25}=\frac{Y_{2,1}^{[3]}}{1+Y_{2,1}^{[3]}}
$$

\section{A.2 Seven-point}

For seven-point case, we have six independent cross ratios. In the practical calculation one can focus on seven consecutive-cusp cross ratios,

$$
u_{i, i+3} \equiv \frac{x_{i, i+2}^{2} x_{i+3, i+6}^{2}}{x_{i, i+3}^{2} x_{i+2, i+6}^{2}}, \quad i=1, \ldots, 7 .
$$

Other cross ratios can be constructed from them directly. They are related to $Y$ functions as

$$
\begin{aligned}
& u_{37}=\frac{Y_{2,2}^{[-2]}}{1+Y_{2,2}^{[-2]}}, \quad u_{14}=\frac{Y_{2,2}}{1+Y_{2,2}}, \quad u_{25}=\frac{Y_{2,2}^{[2]}}{1+Y_{2,2}^{[2]}}, \\
& u_{36}=\frac{Y_{2,1}^{[-3]}}{1+Y_{2,1}^{[-3]}}, \quad u_{47}=\frac{Y_{2,1}^{-}}{1+Y_{2,1}^{-}}, \quad u_{15}=\frac{Y_{2,1}^{+}}{1+Y_{2,1}^{+}}, \quad u_{26}=\frac{Y_{2,1}^{[3]}}{1+Y_{2,1}^{[3]}} \text {. }
\end{aligned}
$$

We mention that there are many other choice to define the cross ratios, for example we also have

$$
u_{37}=\frac{Y_{2,1}^{[5]}}{1+Y_{2,1}^{[5]}}, \quad u_{14}=\frac{Y_{2,1}^{[7]}}{1+Y_{2,1}^{[7]}}, \quad u_{25}=\frac{Y_{2,1}^{[9]}}{1+Y_{2,1}^{[9]}} .
$$

Different definitions are equivalent with each other, due to the relation between $Y$ functions, in particular the periodic relation of $Y$ functions

$$
Y_{2,1}^{[l]}=Y_{2,2}^{[l+7]}, \quad Y_{2, s}^{[l]}=Y_{2, s}^{[l+14]}
$$




\section{A.3 Eight-point}

For eight-point case, we have nine independent cross ratios. We will need the following twelve cross ratios in the calculation

$$
\begin{aligned}
u_{i, i+3} \equiv \frac{x_{i, i+4}^{2} x_{i+1, i+3}^{2}}{x_{i, i+3}^{2} x_{i+1, i+4}^{2}}, & i=1, \ldots, 8, \\
u_{i, i+4} \equiv \frac{x_{i, i+5}^{2} x_{i+1, i+4}^{2}}{x_{i, i+4}^{2} x_{i+1, i+5}^{2}}, & i=1, \ldots, 4 .
\end{aligned}
$$

They are related to $Y$ functions as

$$
\begin{aligned}
& u_{48}=\frac{Y_{2,2}^{[-2]}}{1+Y_{2,2}^{[-2]}}, \quad u_{15}=\frac{Y_{2,2}}{1+Y_{2,2}}, \quad u_{26}=\frac{Y_{2,2}^{[2]}}{1+Y_{2,2}^{[2]}}, \quad u_{37}=\frac{Y_{2,2}^{[4]}}{1+Y_{2,2}^{[4]}}, \\
& u_{47}=\frac{Y_{2,1}^{[-3]}}{1+Y_{2,1}^{[-3]}}, \quad u_{58}=\frac{Y_{2,1}^{-}}{1+Y_{2,1}^{-}}, \quad u_{16}=\frac{Y_{2,1}^{+}}{1+Y_{2,1}^{+}}, \quad u_{27}=\frac{Y_{2,1}^{[3]}}{1+Y_{2,1}^{[3]}} \text {, } \\
& u_{38}=\frac{Y_{2,3}^{[-3]}}{1+Y_{2,3}^{[-3]}}, \quad u_{14}=\frac{Y_{2,3}^{-}}{1+Y_{2,1}^{-}}, \quad u_{25}=\frac{Y_{2,3}^{+}}{1+Y_{2,3}^{+}}, \quad u_{36}=\frac{Y_{2,3}^{[3]}}{1+Y_{2,3}^{[3]}} .
\end{aligned}
$$

In this case, we have that

$$
Y_{2,1}^{[l]}=Y_{2,3}^{[l+8]}, \quad Y_{2,2}^{[l]}=Y_{2,2}^{[l+8]}, \quad Y_{2, s}^{[l]}=Y_{2, s}^{[l+16]} .
$$

\section{A.4 Nine-point}

For nine-point case, we have twelve independent cross ratios. We will need the following 18 cross ratios,

$$
\begin{aligned}
u_{i, i+3} \equiv \frac{x_{i, i+4}^{2} x_{i+1, i+3}^{2}}{x_{i, i+3}^{2} x_{i+1, i+4}^{2}}, & i=1, \ldots, 9, \\
u_{i, i+4} \equiv \frac{x_{i, i+5}^{2} x_{i+1, i+4}^{2}}{x_{i, i+4}^{2} x_{i+1, i+5}^{2}}, & i=1, \ldots, 9 .
\end{aligned}
$$

They are related to $Y$ functions as

$$
\begin{aligned}
& u_{48}=\frac{Y_{2,2}^{[-4]}}{1+Y_{2,2}^{[-4]}}, \quad u_{59}=\frac{Y_{2,2}^{[-2]}}{1+Y_{2,2}^{[-2]}}, \quad u_{16}=\frac{Y_{2,2}}{1+Y_{2,2}}, \quad u_{27}=\frac{Y_{2,2}^{[2]}}{1+Y_{2,2}^{[2]}}, \quad u_{38}=\frac{Y_{2,2}^{[4]}}{1+Y_{2,2}^{[4]}}, \\
& u_{39}=\frac{Y_{2,4}^{[-4]}}{1+Y_{2,4}^{[-4]}}, \quad u_{14}=\frac{Y_{2,4}^{[-2]}}{1+Y_{2,4}^{[-2]}}, \quad u_{25}=\frac{Y_{2,4}}{1+Y_{2,4}}, \quad u_{36}=\frac{Y_{2,4}^{[2]}}{1+Y_{2,4}^{[2]}}, \quad u_{47}=\frac{Y_{2,4}^{[4]}}{1+Y_{2,4}^{[4]}}, \\
& u_{58}=\frac{Y_{2,1}^{[-3]}}{1+Y_{2,1}^{[-3]}}, \quad u_{69}=\frac{Y_{2,1}^{-}}{1+Y_{2,1}^{-}}, \quad u_{17}=\frac{Y_{2,1}^{+}}{1+Y_{2,1}^{+}}, \quad u_{28}=\frac{Y_{2,1}^{[3]}}{1+Y_{2,1}^{[3]}}, \\
& u_{49}=\frac{Y_{2,3}^{[-3]}}{1+Y_{2,3}^{[-3]}}, \quad u_{15}=\frac{Y_{2,3}^{-}}{1+Y_{2,3}^{-}}, \quad u_{26}=\frac{Y_{2,3}^{+}}{1+Y_{2,3}^{+}}, \quad u_{37}=\frac{Y_{2,3}^{[3]}}{1+Y_{2,3}^{[3]}} .
\end{aligned}
$$

In this case, we have that

$$
Y_{2,1}^{[l]}=Y_{2,4}^{[l+9]}, \quad Y_{2,2}^{[l]}=Y_{2,3}^{[l+9]}, \quad Y_{2, s}^{[l]}=Y_{2, s}^{[l+18]} .
$$




\section{B $Y$ and $T$ functions in different phase regions}

To obtain the traditional cross ratios, we need to consider a set of $Y$ functions in deferent phase regions, $Y_{a, s}^{[k]}(i \phi)$. We need to modify the integral equations and add pole contributions from the kernels while crossing the lines of $\pm i \pi / 4, \pm i \pi / 2$ and so on. In this appendix, we give some explicit formulas which are used in the calculation of this paper. We take $m_{s}$ to be real positive, and $\operatorname{Im}(\theta) \in(0, \pi / 4)$. We use the convention that

$$
f^{[l]}(\theta)=f(\theta+i l \pi / 4)
$$

We first define functions for all range of $\theta$

$$
\begin{aligned}
& F_{2, s}(\theta) \equiv-\sqrt{2} m_{s} \cosh (\theta)-K_{2} \star \alpha_{s}-K_{1} \star \beta_{s}, \\
& F_{1, s}(\theta) \equiv-m_{s} \cosh (\theta)-C_{s}-\frac{1}{2} K_{2} \star \beta_{s}-K_{1} \star \alpha_{s}-\frac{1}{2} K_{3} \star \gamma_{s}, \\
& F_{3, s}(\theta) \equiv-m_{s} \cosh (\theta)+C_{s}-\frac{1}{2} K_{2} \star \beta_{s}-K_{1} \star \alpha_{s}+\frac{1}{2} K_{3} \star \gamma_{s} .
\end{aligned}
$$

where $\alpha, \beta, \gamma$ and kernels are given in (21), (22). We emphasize that the $Y$ functions with a given integral form are only defined in a given phase region of $\theta$. The $Y$ functions in general phase regions can be given by including various pole contributions as

$$
\begin{aligned}
\log Y_{2, s}(\theta) & =F_{2, s}(\theta) \\
\log Y_{2, s}^{[1]}(\theta) & =F_{2, s}^{[1]}(\theta)-\alpha_{s}(\theta) \\
\log Y_{2, s}^{[2]}(\theta) & =F_{2, s}^{[2]}(\theta)-\alpha_{s}^{[1]}(\theta)-\beta_{s}(\theta) \\
\log Y_{2, s}^{[3]}(\theta) & =F_{2, s}^{[3]}(\theta)-\alpha_{s}^{[2]}(\theta)-\beta_{s}^{[1]}(\theta)-\alpha_{s}(\theta) \\
\log Y_{2, s}^{[4]}(\theta) & =F_{2, s}^{[4]}(\theta)-\alpha_{s}^{[3]}(\theta)-\beta_{s}^{[2]}(\theta)-\alpha_{s}^{[1]}(\theta) \\
\log Y_{2, s}^{[-1]}(\theta) & =F_{2, s}^{[-1]}(\theta) \\
\log Y_{2, s}^{[-2]}(\theta) & =F_{2, s}^{[-2]}(\theta)-\alpha_{s}^{[-1]}(\theta) \\
\log Y_{2, s}^{[-3]}(\theta) & =F_{2, s}^{[-3]}(\theta)-\alpha_{s}^{[-2]}(\theta)-\beta_{s}^{[-1]}(\theta) \\
\log Y_{2, s}^{[-4]}(\theta) & =F_{2, s}^{[-4]}(\theta)-\alpha_{s}^{[-3]}(\theta)-\beta_{s}^{[-2]}(\theta)-\alpha_{s}^{[-1]}(\theta) \\
\log Y_{1, s}(\theta) & =F_{1, s}(\theta) \\
\log Y_{1, s}^{[1]}(\theta) & =F_{1, s}^{[1]}(\theta)-\frac{1}{2} \beta_{s}(\theta)+\frac{1}{2} \gamma_{s}(\theta) \\
\log Y_{1, s}^{[2]}(\theta) & =F_{1, s}^{[2]}(\theta)-\frac{1}{2} \beta_{s}^{[1]}(\theta)+\frac{1}{2} \gamma_{s}^{[1]}(\theta)-\alpha_{s}(\theta) \\
\log Y_{1, s}^{[3]}(\theta) & =F_{1, s}^{[3]}(\theta)-\frac{1}{2} \beta_{s}^{[2]}(\theta)+\frac{1}{2} \gamma_{s}^{[2]}(\theta)-\alpha_{s}^{[1]}(\theta)-\frac{1}{2} \beta_{s}(\theta)+\frac{1}{2} \gamma_{s}(\theta) \\
\log Y_{1, s}^{[4]}(\theta) & =F_{1, s}^{[4]}(\theta)-\frac{1}{2} \beta_{s}^{[3]}(\theta)+\frac{1}{2} \gamma_{s}^{[3]}(\theta)-\alpha_{s}^{[2]}(\theta)-\frac{1}{2} \beta_{s}^{[1]}(\theta)+\frac{1}{2} \gamma_{s}^{[1]}(\theta) \\
\log Y_{1, s}^{[-1]}(\theta) & =F_{1, s}^{[-1]}(\theta) \\
\log Y_{1, s}^{[-2]}(\theta) & =F_{1, s}^{[-2]}(\theta)-\frac{1}{2} \beta_{s}^{[-1]}(\theta)-\frac{1}{2} \gamma_{s}^{[-1]}(\theta)
\end{aligned}
$$




$$
\begin{aligned}
& \log Y_{1, s}^{[-3]}(\theta)=F_{1, s}^{[-3]}(\theta)-\frac{1}{2} \beta_{s}^{[-2]}(\theta)-\frac{1}{2} \gamma_{s}^{[-2]}(\theta)-\alpha_{s}^{[-1]}(\theta), \\
& \log Y_{1, s}^{[-4]}(\theta)=F_{1, s}^{[-4]}(\theta)-\frac{1}{2} \beta_{s}^{[-3]}(\theta)-\frac{1}{2} \gamma_{s}^{[-3]}(\theta)-\alpha_{s}^{[-2]}(\theta)-\frac{1}{2} \beta_{s}^{[-1]}(\theta)-\frac{1}{2} \gamma_{s}^{[-1]}(\theta),
\end{aligned}
$$

and similar for $Y_{3, s}^{[k]}$ with only the sign for the $\gamma_{s}^{[r]}$ terms changed compared to $Y_{1, s}^{[k]}$.

For the calculation of extra parts, we also list the formulas of $T_{2,1}$ and $T_{2,2}$ that are given in (24) and (27). Similarly, we first define functions for all range of $\theta$

$$
\begin{aligned}
& G_{2,1}(\theta) \equiv K_{2} \star \hat{\beta}_{1}+K_{1} \star \hat{\alpha}_{1}, \\
& G_{2,2}(\theta) \equiv-\sqrt{2} m_{1} \cosh (\theta)+K_{2} \star \hat{\beta}_{2}+K_{1} \star \hat{\alpha}_{2},
\end{aligned}
$$

where

$$
\hat{\beta}_{s} \equiv \log \left(1+Y_{2, s}\right), \quad \hat{\alpha}_{s} \equiv \log \left(1+Y_{1, s}\right)\left(1+Y_{3, s}\right) .
$$

Then we have $T$ functions in general phase regions as

$$
\begin{aligned}
\log T_{2, s}(\theta) & =G_{2, s}(\theta) \\
\log T_{2, s}^{[1]}(\theta) & =G_{2, s}^{[1]}(\theta)-\hat{\beta}_{s}(\theta) \\
\log T_{2, s}^{[2]}(\theta) & =G_{2, s}^{[2]}(\theta)-\hat{\beta}_{s}^{[1]}(\theta)-\hat{\alpha}_{s}(\theta) \\
\log T_{2, s}^{[3]}(\theta) & =G_{2, s}^{[3]}(\theta)-\hat{\beta}_{s}^{[2]}(\theta)-\hat{\alpha}_{s}^{[1]}(\theta)-\hat{\beta}_{s}(\theta) \\
\log T_{2, s}^{[4]}(\theta) & =G_{2, s}^{[4]}(\theta)-\hat{\beta}_{s}^{[3]}(\theta)-\hat{\alpha}_{s}^{[2]}(\theta)-\hat{\beta}_{s}^{[1]}(\theta), \\
\log T_{2, s}^{[-1]}(\theta) & =G_{2, s}^{[-1]}(\theta) \\
\log T_{2, s}^{[-2]}(\theta) & =G_{2, s}^{[-2]}(\theta)-\hat{\beta}_{s}^{[-1]}(\theta) \\
\log T_{2, s}^{[-3]}(\theta) & =G_{2, s}^{[-3]}(\theta)-\hat{\beta}_{s}^{[-2]}(\theta)-\hat{\alpha}_{s}^{[-1]}(\theta) \\
\log T_{2, s}^{[-4]}(\theta) & =G_{2, s}^{[-4]}(\theta)-\hat{\beta}_{s}^{[-3]}(\theta)-\hat{\alpha}_{s}^{[-2]}(\theta)-\hat{\beta}_{s}^{[-1]}(\theta) .
\end{aligned}
$$

\section{Results of amplitudes and remainder functions}

We list the explicit results of amplitudes up to nine points in this appendix. $A_{\mathrm{BDS}}$ is the one-loop finite part of amplitudes at weak couping [12]. The result of $A_{\mathrm{BDS}-\text { like }}, A_{\text {periods }}$ and $A_{\text {free }}$ for $n \neq 4 K$ cases were given in [25]. $A_{\text {extra }}$ and $A_{\mathrm{BDS}-\text { like }}$ for eight-point were given in [27]. The periods part of eight-point are calculated in this paper. We also give the result of the difference between BDS-like

and BDS part in terms of cross ratios beyond six-point for the first time. The total amplitudes and remainder functions can be constructed as

$$
\begin{aligned}
& A=A_{\text {div }}+A_{\text {BDS-like }}+A_{\text {extra }}+A_{\text {periods }}+A_{\text {free }}, \\
& R=\left(A_{\text {BDS-like }}-A_{\mathrm{BDS}}\right)+A_{\text {extra }}+A_{\text {periods }}+A_{\text {free }} .
\end{aligned}
$$

The IR divergent parts are universal

$$
A_{\mathrm{div}}=\frac{1}{2} \sum_{i=1}^{n}\left(L+\frac{\ell_{i}}{2}\right)^{2},
$$

where $L$ is the cutoff for the minimal surface. We use the notation that

$$
\ell_{i} \equiv \log x_{i, i+2}^{2}, \quad \ell_{i j} \equiv \log x_{i j}^{2} .
$$




\section{C.1 Six-point}

$$
\begin{aligned}
& A_{\text {periods }}=\frac{\left|m_{1}\right|^{2}}{4} \\
& A_{\mathrm{BDS}-\text { like }}=-\frac{1}{8} \sum_{i=1}^{6}\left(\ell_{i}^{2}+\sum_{k=0}^{2} \ell_{i} \ell_{i+2 k+1}(-1)^{k+1}\right) \\
& A_{\text {free }}=\frac{\left|m_{1}\right|}{2 \pi} \int_{-\infty}^{+\infty} d \theta \cosh \theta \log \left[\left(1+Y_{1,1}\right)\left(1+Y_{3,1}\right)\left(1+Y_{2,1}\right)^{\sqrt{2}}\right] . \\
& A_{\mathrm{BDS}-\text { like }}-A_{\mathrm{BDS}}=-\sum_{i=1}^{3}\left(\frac{1}{8} \log ^{2} u_{i}+\frac{1}{4} L i_{2}\left(1-u_{i}\right)\right) .
\end{aligned}
$$

\section{C.2 Seven-point}

$$
\begin{gathered}
A_{\text {BDS-like }}=-\frac{1}{4} \sum_{i=1}^{7}\left(\ell_{i}^{2}+\sum_{k=0}^{2} \ell_{i} \ell_{i+2 k+1}(-1)^{k+1}\right) \\
A_{\text {periods }}=\frac{\left|m_{1}\right|^{2}+\left|m_{2}\right|^{2}}{2}+\frac{m_{1} \bar{m}_{2}+\bar{m}_{1} m_{2}}{2 \sqrt{2}}, \\
A_{\text {free }}=\sum_{s=1}^{2} \frac{\left|m_{s}\right|}{2 \pi} \int_{-\infty}^{+\infty} d \theta \cosh \theta \log \left[\left(1+Y_{1, s}\right)\left(1+Y_{3, s}\right)\left(1+Y_{2, s}\right)^{\sqrt{2}}\right] . \\
A_{\mathrm{BDS}-\text { like }}-A_{\mathrm{BDS}}=-\frac{1}{4} \sum_{i=1}^{7}\left(\log ^{2} u_{i, i+3}+L i_{2}\left(1-u_{i, i+3}\right)-\frac{1}{2} \log u_{i, i+3} \log \frac{u_{i+2, i+5} u_{i+1, i+5}}{u_{i+3, i+6} u_{i, i+4}}\right) .
\end{gathered}
$$

\section{C.3 Eight-point}

$$
\begin{aligned}
& A_{\text {periods }}=\frac{\left|m_{2}\right|^{2}+\left|m_{3}\right|^{2}}{2}+\frac{m_{2} \bar{m}_{3}+\bar{m}_{2} m_{3}}{2 \sqrt{2}}-\frac{1}{4}\left|m_{1}+\sqrt{2} m_{2}+m_{3}\right|^{2}, \\
& A_{\text {free }}=\sum_{s=1}^{3} \frac{\left|m_{s}\right|}{2 \pi} \int_{-\infty}^{+\infty} d \theta \cosh \theta \log \left[\left(1+Y_{1, s}\right)\left(1+Y_{3, s}\right)\left(1+Y_{2, s}\right)^{\sqrt{2}}\right], \\
& A_{\text {BDS-like }}=-\frac{1}{8} \sum_{i=1}^{8} \ell_{i}^{2}+\frac{1}{4} \sum_{i=1}^{8} \ell_{i} \ell_{i+1}-\frac{1}{4}\left(\ell_{2}+\ell_{6}\right)\left(\ell_{3}+\ell_{7}\right)+\frac{1}{4}\left(\Delta_{x} \ell_{48}-\Delta_{y} \ell_{37}\right), \\
& A_{\text {extra }}=-\frac{1}{4}\left(\Delta_{x} \log T_{2,2}^{[-3]}-\Delta_{y} \log T_{2,2}^{[-5]}\right),
\end{aligned}
$$

where

$$
\Delta_{x}=-\ell_{1}+\ell_{3}-\ell_{5}+\ell_{7}, \quad \Delta_{y}=-\ell_{2}+\ell_{4}-\ell_{6}+\ell_{8} .
$$




$$
\begin{aligned}
A_{\mathrm{BDS}-\mathrm{like}}-A_{\mathrm{BDS}}= & -\frac{1}{8} \log ^{2}\left(\frac{u_{14} u_{25} u_{58} u_{16}}{u_{36} u_{47} u_{27}}\right)-\frac{1}{4} \log \left(u_{15} u_{16} u_{25} u_{26}\right) \log \left(u_{26} u_{27} u_{36} u_{37}\right) \\
& -\frac{1}{2} \log \left(\frac{u_{15}}{u_{37}}\right) \log \left(\frac{u_{14} u_{25} u_{58} u_{16} u_{15}}{u_{36} u_{47} u_{27} u_{37}}\right)-\frac{1}{8}\left(\Delta_{x}-\Delta_{y}\right)^{2} \\
& +\frac{1}{4} \Delta_{x} \log \left(\frac{u_{14} u_{15}^{2} u_{16} u_{25} u_{58}}{u_{27} u_{36} u_{37}^{2} u_{47}}\right)+\frac{1}{4} \Delta_{y} \log \left(\frac{u_{26} u_{27} u_{36} u_{37}^{2} u_{47}}{u_{14} u_{15} u_{58}}\right) \\
& -\frac{1}{8} \sum_{i=1}^{8} \log ^{2} u_{i, i+3}-\frac{1}{8} \sum_{i=1}^{8}\left[2 L i_{2}\left(1-u_{i, i+3}\right)+L i_{2}\left(1-u_{i, i+4}\right)\right] .
\end{aligned}
$$

\section{C.4 Nine-point}

$$
\begin{gathered}
A_{\mathrm{BDS}-\mathrm{like}}=-\frac{1}{4} \sum_{i=1}^{9}\left(\ell_{i}^{2}+\sum_{k=0}^{4} \ell_{i} \ell_{i+1+2 k}(-1)^{k+1}\right), \\
A_{\text {free }}=\sum_{s=1}^{4} \frac{\left|m_{s}\right|}{2 \pi} \int_{-\infty}^{+\infty} d \theta \cosh \theta \log \left[\left(1+Y_{1, s}\right)\left(1+Y_{3, s}\right)\left(1+Y_{2, s}\right)^{\sqrt{2}}\right] \\
A_{\text {periods }}=-\frac{1}{2}\left(\left|m_{2}\right|^{2}+\left|m_{3}\right|^{2}+m_{1} \bar{m}_{3}+m_{3} \bar{m}_{1}+m_{2} \bar{m}_{4}+m_{4} \bar{m}_{2}\right) \\
-\frac{\sqrt{2}}{4}\left(m_{1} \bar{m}_{2}+m_{2} \bar{m}_{1}+m_{1} \bar{m}_{4}+m_{4} \bar{m}_{1}+m_{3} \bar{m}_{4}+m_{4} \bar{m}_{3}+2 m_{2} \bar{m}_{3}+2 m_{3} \bar{m}_{2}\right) . \\
A_{\mathrm{BDS}-\mathrm{like}}-A_{\mathrm{BDS}}=\frac{1}{4} \sum_{i=1}^{9}\left[\log u_{i, i+3} \log \left(\frac{u_{i, i+4} u_{i+4, i+7}}{u_{i+1, i+5} u_{i+2, i+5} u_{i+3, i+6} u_{i+2, i+6}^{2}}\right)\right. \\
\left.+\log u_{i, i+4} \log \left(\frac{u_{i, i+4} u_{i+1, i+4} u_{i+4, i+8}^{2} u_{i+5, i+8}^{2}}{u_{i+2, i+5} u_{i+2, i+6}^{3} u_{i+3, i+6}^{2} u_{i+3, i+7}^{2}}\right)-L i_{2}\left(1-u_{i, i+3}\right)-L i_{2}\left(1-u_{i, i+4}\right)\right] .
\end{gathered}
$$

\section{References}

[1] L. F. Alday and J. M. Maldacena, "Gluon scattering amplitudes at strong coupling," JHEP 0706, 064 (2007) arXiv:0705.0303 [hep-th]].

[2] J. M. Drummond, G. P. Korchemsky and E. Sokatchev, "Conformal properties of four-gluon planar amplitudes and Wilson loops," Nucl. Phys. B 795, 385 (2008) arXiv:0707.0243 [hepth]]. J. M. Drummond, J. Henn, G. P. Korchemsky and E. Sokatchev, "On planar gluon amplitudes/Wilson loops duality," Nucl. Phys. B 795, 52 (2008) [arXiv:0709.2368 [hep-th]].

[3] A. Brandhuber, P. Heslop and G. Travaglini, "MHV Amplitudes in N=4 Super Yang-Mills and Wilson Loops," Nucl. Phys. B 794, 231 (2008) [arXiv:0707.1153 [hep-th]].

[4] J. M. Drummond, J. Henn, G. P. Korchemsky and E. Sokatchev, "Conformal Ward identities for Wilson loops and a test of the duality with gluon amplitudes," Nucl. Phys. B 826, 337 (2010) arXiv:0712.1223 [hep-th]].

[5] J. M. Drummond, J. Henn, G. P. Korchemsky and E. Sokatchev, "Dual superconformal symmetry of scattering amplitudes in $\mathrm{N}=4$ super-Yang-Mills theory," Nucl. Phys. B 828, 317 (2010) arXiv:0807.1095 [hep-th]]. 
[6] N. Berkovits and J. Maldacena, "Fermionic T-Duality, Dual Superconformal Symmetry, and the Amplitude/Wilson Loop Connection," JHEP 0809, 062 (2008) [arXiv:0807.3196[hep-th]].

[7] N. Beisert, R. Ricci, A. A. Tseytlin and M. Wolf, "Dual Superconformal Symmetry from AdS5 x S5 Superstring Integrability," Phys. Rev. D 78, 126004 (2008) [arXiv:0807.3228 [hep-th]].

Mandal:2002fs,Minahan:2002ve,Bena:2003wd

[8] G. Mandal, N. V. Suryanarayana and S. R. Wadia, "Aspects of semiclassical strings in AdS(5)," Phys. Lett. B 543, 81 (2002) arXiv:hep-th/0206103.

[9] J. A. Minahan and K. Zarembo, "The Bethe-ansatz for N $=4$ super Yang-Mills," JHEP 0303, 013 (2003) arXiv:hep-th/0212208.

[10] I. Bena, J. Polchinski and R. Roiban, "Hidden symmetries of the AdS(5) x S**5 superstring," Phys. Rev. D 69, 046002 (2004) arXiv:hep-th/0305116.

[11] J. M. Drummond, J. M. Henn and J. Plefka, JHEP 0905, 046 (2009) arXiv:0902.2987 [hepth]].

[12] Z. Bern, L. J. Dixon and V. A. Smirnov, "Iteration of planar amplitudes in maximally supersymmetric Yang-Mills theory at three loops and beyond," Phys. Rev. D 72, 085001 (2005) arXiv:hep-th/0505205.

[13] C. Anastasiou, Z. Bern, L. J. Dixon and D. A. Kosower, "Planar amplitudes in maximally supersymmetric Yang-Mills theory," Phys. Rev. Lett. 91, 251602 (2003) arXiv:hep-th/0309040.

[14] Z. Bern, M. Czakon, D. A. Kosower, R. Roiban and V. A. Smirnov, "Two-loop iteration of five-point N = 4 super-Yang-Mills amplitudes," Phys. Rev. Lett. 97, 181601 (2006) arXiv:hep-th/0604074. Z. Bern, M. Czakon, L. J. Dixon, D. A. Kosower and V. A. Smirnov, “ The Four-Loop Planar Amplitude and Cusp Anomalous Dimension in Maximally Supersymmetric Yang-Mills Theory," Phys. Rev. D 75 (2007) 085010, arXiv:hep-th/0610248. Z. Bern, J. J. M. Carrasco, H. Johansson and D. A. Kosower, " Maximally supersymmetric planar Yang-Mills amplitudes at five loops," arXiv:0705.1864 [hep-th].

[15] F. Cachazo, M. Spradlin and A. Volovich, "Four-Loop Cusp Anomalous Dimension From Obstructions," Phys. Rev. D 75, 105011 (2007) arXiv:hep-th/0612309.

[16] L. F. Alday and J. Maldacena, "Comments on gluon scattering amplitudes via AdS/CFT," JHEP 0711, 068 (2007) arXiv:0710.1060 [hep-th]].

[17] Z. Bern, L. J. Dixon, D. A. Kosower, R. Roiban, M. Spradlin, C. Vergu and A. Volovich, "The Two-Loop Six-Gluon MHV Amplitude in Maximally Supersymmetric Yang-Mills Theory," Phys. Rev. D 78, 045007 (2008) arXiv:0803.1465 [hep-th]].

[18] J. M. Drummond, J. Henn, G. P. Korchemsky and E. Sokatchev, "Hexagon Wilson loop = six-gluon MHV amplitude," Nucl. Phys. B 815, 142 (2009) [arXiv:0803.1466 [hep-th]].

[19] C. Anastasiou, A. Brandhuber, P. Heslop, V. V. Khoze, B. Spence and G. Travaglini, "TwoLoop Polygon Wilson Loops in N=4 SYM," JHEP 0905, 115 (2009) arXiv:0902.2245 [hepth]]. 
[20] A. Brandhuber, P. Heslop, V. V. Khoze and G. Travaglini, "Simplicity of Polygon Wilson Loops in N=4 SYM," JHEP 1001, 050 (2010) [arXiv:0910.4898]. P. Heslop and V. V. Khoze, "Regular Wilson loops and MHV amplitudes at weak and strong coupling," arXiv:1003.4405.

[21] V. Del Duca, C. Duhr and V. A. Smirnov, "The Two-Loop Hexagon Wilson Loop in N $=4$ SYM," arXiv:1003.1702.

[22] J. H. Zhang, "On the two-loop hexagon Wilson loop remainder function in N=4 SYM," arXiv:1004.1606 [hep-th].

[23] L. F. Alday and J. Maldacena, "Null polygonal Wilson loops and minimal surfaces in Antide-Sitter space," JHEP 0911, 082 (2009) [arXiv:0904.0663 [hep-th]].

[24] L. F. Alday, D. Gaiotto and J. Maldacena, "Thermodynamic Bubble Ansatz," arXiv:0911.4708 [hep-th].

[25] L. F. Alday, J. Maldacena, A. Sever and P. Vieira, "Y-system for Scattering Amplitudes," arXiv:1002.2459 [hep-th].

[26] L. F. Alday, D. Gaiotto, J. Maldacena, A. Sever and P. Vieira, "An Operator Product Expansion for Polygonal null Wilson Loops," arXiv:1006.2788 [hep-th].

[27] G. Yang, "Scattering amplitudes at strong coupling for 4K gluons," arXiv:1004.3983 [hep-th].

[28] C. N. Yang and C. P. Yang, "Thermodynamics of a one-dimensional system of bosons with repulsive delta-function interaction," J. Math. Phys. 10 (1969) 1115.

[29] A. B. Zamolodchikov, "Thermodynamic Bethe ansatz in relativistic models. Scaling three state Potts and Lee-Yang Models," Nucl. Phys. B 342, 695 (1990).

[30] D. Gaiotto, G. W. Moore and A. Neitzke, "Four-dimensional wall-crossing via threedimensional field theory," arXiv:0807.4723 [hep-th].

[31] D. Gaiotto, G. W. Moore and A. Neitzke, "Wall-crossing, Hitchin Systems, and the WKB Approximation," arXiv:0907.3987 [hep-th].

[32] Z. Bern and G. Chalmers, "Factorization in one loop gauge theory," Nucl. Phys. B 447, 465 (1995) arXiv:hep-ph/9503236.

[33] A. Hodges, "Eliminating spurious poles from gauge-theoretic amplitudes," arXiv:0905.1473 [hep-th].

[34] L. Mason and D. Skinner, "Dual Superconformal Invariance, Momentum Twistors and Grassmannians," JHEP 0911, 045 (2009) [arXiv:0909.0250 [hep-th]].

[35] Y. Hatsuda, K. Ito, K. Sakai and Y. Satoh, "Six-point gluon scattering amplitudes from $Z_{4}$-symmetric integrable model," arXiv:1005.4487 [hep-th]. 